\title{
Structure of the Microemulsion of Polyglycerol Polyricinoleate Encapsulating Vitamin E
}

\author{
Junji Matsuoka', Takumi Kusano', Yuuki Kasama², Etsuko Tominaga ${ }^{2}$, Junya Kobayashi², \\ Wataru Fujii ${ }^{2}$, Hiroki Iwase ${ }^{3}$, Mitsuhiro Shibayama ${ }^{1 *}$ and Hironobu Nanbu ${ }^{2 *}$ \\ ${ }^{1}$ Institute for Solid State Physics, The University of Tokyo, 5-1-5 Kashiwanoha, Kashiwa, Chiba 277-8581, JAPAN \\ ${ }^{2}$ Taiyo Kagaku Co., Ltd., 1-3 Takara-machi, Yokkaichi, Mie 510-0844, JAPAN \\ ${ }^{3}$ Neutron Science and Technology Center, CROSS, 162-1 Tokai, Ibaraki 319-1106, JAPAN
}

\begin{abstract}
The structures of micelles and microemulsions consisting of polyglycerol polyricinoleate (PGPR) were investigated by small-angle X-ray scattering (SAXS) and rheological measurements. The SAXS results show that amphiphilic PGPR molecules form stable micelles in glycerol. When vitamin $E$ is added to the PGPR micelles, it is encapsulated in the micelles and forms an emulsion. These micelles are stable towards mechanical shearing up to a shear rate of $1000 \mathrm{~s}^{-1}$, with shear thinning occurring in the emulsion above 100 $\mathrm{s}^{-1}$, indicating that the emulsion may undergo break up by shearing, but recovers the structure by releasing shear strain.
\end{abstract}

Key words: microemulsion, polyglycerol polyricinoleate, rheology, small angle X-ray scattering, vitamin E

\section{Introduction}

Research into microemulsions, micelles, vesicles, and liposomes with appropriate surfactants, lipids, and amphiphiles are important for the fundamental understanding of emulsion properties. Basic assemblies and micro/nanostructures have received increasing attention in recent studies in the field of nanoscience and nanotechnology ${ }^{1-4)}$. Microemulsions are thermodynamically stable, transparent, isotropic solutions with particle sizes ranging from 5 to 100 $\mathrm{nm}$. They are formed by the spontaneous self-assembly of hydrophobic and hydrophilic parts of surfactant molecules. In contrast to macroemulsions, made by mechanical stirring, which are thermodynamically unstable, microemulsions have a wide range of applications, including pharmaceutical, cosmetics, oil recovery, and reaction media ${ }^{5}$. For applications of microemulsions in foods, however, many surfactants are not permissible. More importantly, solubilization of long-chain triglycerides (LCT), such as soybean oil, is more difficult to achieve than for lipids those with short or medium length triglycerides. Polyglyceryl esters (PGEs) are important non-ionic surfactants with various applications in the cosmetic, food, and pharmaceutical industries $^{6)}$. The defining feature of PGEs is a large hydrophilic group in the molecule. They have been known since the beginning of the twentieth century and their amphiphilic character enables their use in the stabilization of various suspensions. In cosmetics, they are used to emulsify, control viscosity, disperse and stabilize the final cosmetic mixture. They are incorporated into hair styling gels, skin treatment gels, skin cleansers, baby creams, long-acting hand creams, moisturizing sunscreens, and sun-protective sticks. PGEs have also been used as food additives for many years. They are used as emulsifying agents in the production of fine bakery items, chewing gum, and for the replacement of fats. Furthermore, they are biodegradable, biocompatible, and free from harmful ethylene oxide and nitrosamines.

Certain types of PGE emulsifiers, whose hydrophilic group is extended by the polymerization of glycerol (the average number of polymerization being 2-10), are commonly used in food industry. By changing the chain lengths of hydrophilic and hydrophobic (lipophilic) groups, it is possible to control the physicochemical properties of the surfactants ${ }^{7)}$. Therefore, PGE emulsifiers can be adapted for various systems. The emulsifiers with ricinoleic acid as a lipophilic group are called polyglycerol polyricinoleate $(\mathrm{PGPR})^{8)}$. The length of lipophilic group can be easily controlled using ricinoleic acid because ricinoleic acid can be condensed by esterification. The emulsion formed by PGPR has high environmental stability ${ }^{7}$ and is expected to have high bioabsorbability ${ }^{9}$. For these reasons, PGPRs are applied widely in food industry. To develop a successful

\footnotetext{
*Correspondence to: Mitsuhiro Shibayama, Institute for Solid State Physics, The University of Tokyo, 5-1-5 Kashiwanoha, Kashiwa, Chiba 277-8581, JAPAN. Hironobu Nanbu, Taiyo Kagaku Co., Ltd., 1-3 Takara-machi, Yokkaichi, Mie 510-0844, JAPAN

E-mail: sibayama@ issp.u-tokyo.ac.jp (MS), hnanbu@taiyokagaku.co.jp (HN)

Accepted July 7, 2017 (received for review June 1, 2017)

Journal of Oleo Science ISSN 1345-8957 print / ISSN 1347-3352 online

http://www.jstage.jst.go.jp/browse/jos/ http://mc.manusriptcentral.com/jjocs
} 
emulsification technique, it is important to investigate the structure of the emulsions. In this study, we investigated the formation of a vitamin $\mathrm{E}$ (compounds containing tocopherol and tocotrienols) emulsion stabilized by PGPR. Stable encapsulation is important in the pharmaceutical and food industries to preserve the antioxidant activity of vitamin $\mathrm{E}^{10)}$. Though there are many reports on microemulsions ${ }^{11)}$, including structural investigations with scattering methods ${ }^{12-14)}$, studies with PGPR are lacking, in spite of their practical applications. In this study, we investigated the formation of a vitamin $\mathrm{E}$ emulsion stabilized by PGPR.

\section{Experimental}

Samples: PGPR was supplied by Taiyo Kagaku Co., $\mathrm{Ltd}^{15)}$., and its chemical structure is shown in Fig. 1a. The average degree of polymerization of the glycerol unit, $n$, is 5 and the average condensation number of ricinoleic acid, $m$, is 2 . PGPR and glycerol were mixed at $70^{\circ} \mathrm{C}$. Vitamin $\mathrm{E}$ (Fig. 1b) and epigallocatechin gallate as an antioxidant were added to the PGPR solution and homogenized at $60^{\circ} \mathrm{C}$. Epigallocatechin gallate was used to improve long term stability of the emulsion. For comparison, a PGPR solution without vitamin $\mathrm{E}$ and epigallocatechin gallate was also prepared in the same way.

Small-angle X-ray scattering (SAXS) and rheological measurements: SAXS measurements were carried out using the SAXS spectrometer installed on the BL03XU and BL19B2 beamlines at SPring-8, Hyogo, Japan. All measurements were performed at room temperature. The absolute intensity calibration was conducted with a glassy carbon ${ }^{16)}$. Viscosity measurements were carried out with a stress control rheometer(MCR-501, Anton Paar, Austria) equipped with a cone plate of $25.0 \mathrm{~mm}$ radius and cone angle of $1^{\circ}$. The sample temperature was maintained at $25.00 \pm 0.03^{\circ} \mathrm{C}$.

(a)<smiles>CCCCCCC(C/C=C\CCCCCCCC(=O)OCC(O)COC)OCC</smiles>

(b)

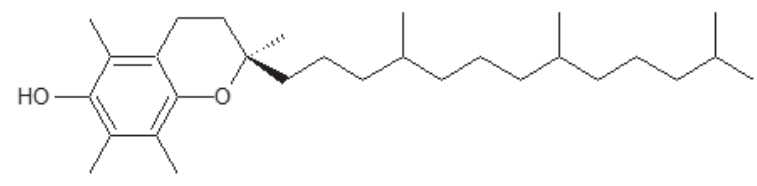

Fig. 1 Chemical structures of (a)PGPR and (b) vitamin E.

\section{Results and Discussion}

\subsection{PGPR micelles}

First, structural analysis of PGPR micelles in glycerol was performed. Figure 2 shows the SAXS intensity functions of PGPR in glycerol. The PGPR concentration, $C_{\mathrm{PGPR}}$, was systematically decreased by dilution with glycerol from 18.35 wt \% to 1.00 wt\%. As shown in Fig. 2, the SAXS intensity increases with increasing $C_{\mathrm{PGPR}}$ and a scattering peak appears for $C_{\mathrm{PGPR}}=15.00$ and $18.35 \mathrm{wt} \%$, indicating some inter-particle interference.

To quantitatively determine the structural parameters of the PGPR micelles, model fitting analysis was performed. First, we estimated the scattering length densities (SLD) of the components. For SAXS measurements, the SLD values of each molecules can be calculated by the following equation:

$$
\rho=\frac{\Sigma b}{V_{m}}=\frac{\Sigma b}{\left(M / N_{A} d\right)}
$$

where $b$ is the scattering length of atoms, $V_{m}$ is the molecular volume, $M$ is the molecular weight, $N_{A}$ is Avogadro's constant, and $d$ is the mass density. For SAXS, $b$ is given by $b=Z \times\left(2.81 \times 10^{-5}\right) \AA(Z$ is the atomic number $)$. Figure 3

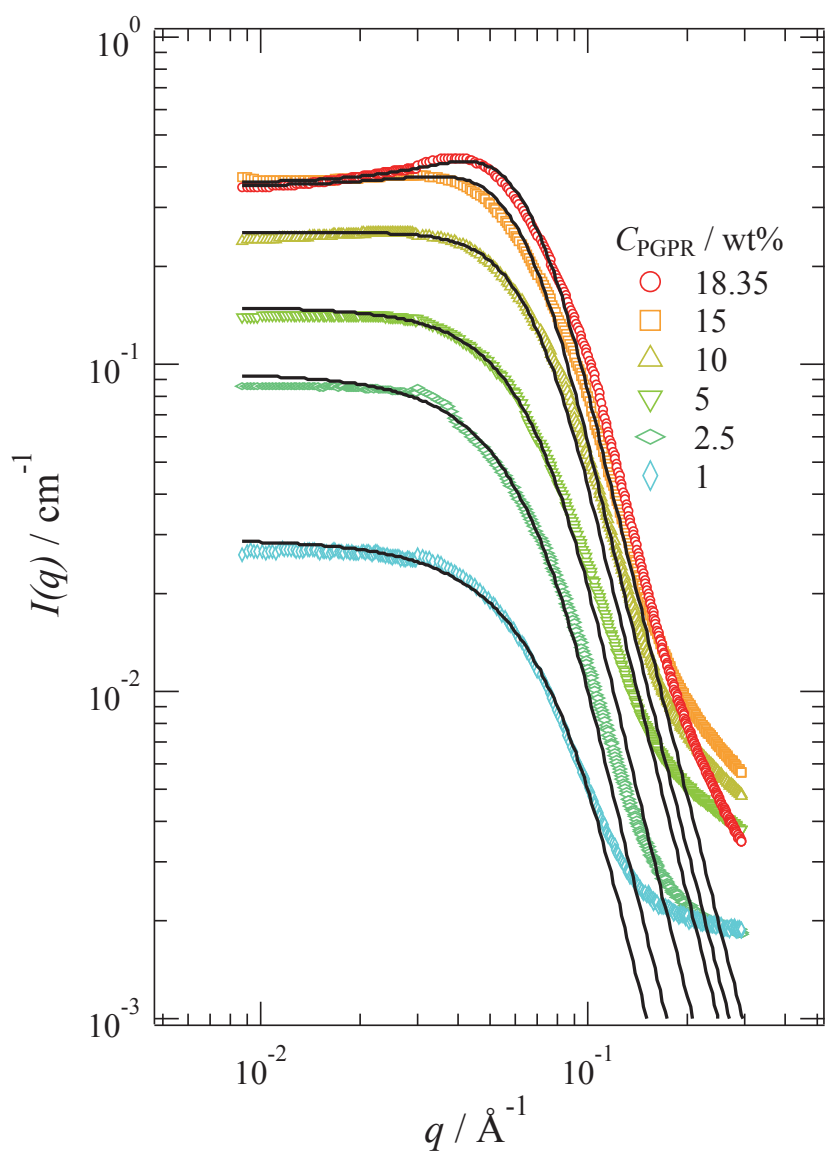

Fig. 2 SAXS curves of PGPR micelles in glycerol at various PGPR concentrations, $C_{\mathrm{PGPR}}$. The solid lines are fitting results with Eqs. (2) - (6). 
shows calculated SLD variations of the PGPR micelles, where the mass densities of the hydrophobic and hydrophilic parts of PGPR were assumed to be the same as those of ricinoleic acid $\left(d_{\mathrm{r}}=0.957 \mathrm{~g} / \mathrm{cm}^{3}\right)$ and of glycerol $\left(d_{\mathrm{g}}=\right.$ $\left.1.261 \mathrm{~g} / \mathrm{cm}^{3}\right)$, respectively. The SLD of the head group of the PGPR molecule is very close to that of glycerol, and we employed a spherical model with box-type SLD variation and carried out curve fitting of the observed SAXS intensity functions.

$$
I(q)=\phi(\Delta \rho)^{2} \frac{\int_{0}^{\bar{R}+3 \sigma} V P_{\mathrm{sph}}(q, R) S_{\mathrm{PY}}(q, R, \phi) \exp \left[-\frac{(R-<R>)^{2}}{2 \sigma^{2}}\right] d R}{\int_{0}^{\bar{R}+3 \sigma} \exp \left[-\frac{(R-<R>)^{2}}{2 \sigma^{2}}\right] d R}
$$

Here $<R>$ and $\sigma$ are the mean value of the radius of the sphere and the standard deviation, respectively. The form factor $P_{\mathrm{sph}}(q, R)$ of the spherical object with the radius $R$ and the volume $V$ is given by

$$
P_{\text {sph }}(q, R)=\frac{9\left[\sin (q R)-q R \cos (q R)^{2}\right]}{(q R)^{6}}
$$

$S_{\mathrm{PY}}(q, R, \phi)$ is the Percus-Yevick structure factor given by ${ }^{17)}$

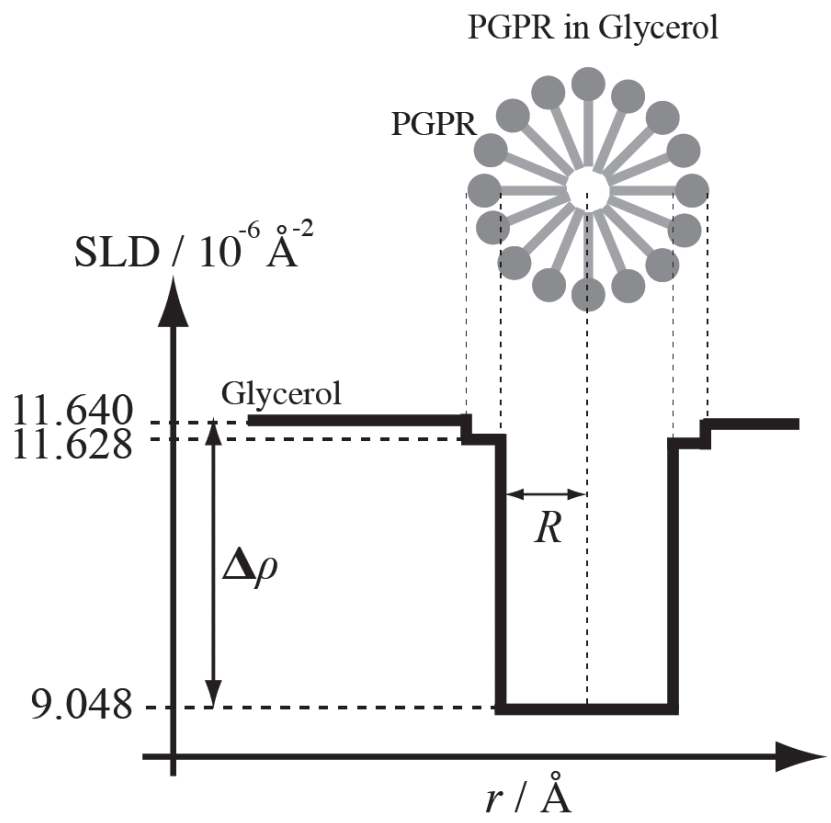

Fig. 3 The calculated scattering length density profile of PGPR micelle in glycerol.

$$
S_{\mathrm{PY}}(q)=\frac{1}{1+24 \phi\left[H_{\mathrm{PY}}(A) / A\right]}
$$

where $\phi$ is the volume fraction of the hard sphere, and $A=$ $2 q R$. The $H_{\mathrm{PY}}$ function is given by the following:

$$
\begin{aligned}
& H_{\mathrm{PY}}(A)=\frac{\alpha}{A^{2}}(\sin A-A \cos A)+\frac{\beta}{A^{3}}[2 A \sin A+(2-A) \cos A-2] \\
& +\frac{\gamma}{A^{5}}\left\{-A^{4} \cos A+4\left[\left(3 A^{2}-6\right) \cos A+\left(A^{3}-6 A\right) \sin A+6\right]\right\} \\
& \alpha=\frac{(1+2 \phi)^{2}}{(1-\phi)^{4}}, \quad \beta=\frac{6 \phi(1+\phi / 2)^{2}}{(1-\phi)^{4}}, \quad \gamma=\frac{\phi(1+2 \phi)^{2}}{2(1-\phi)^{4}}
\end{aligned}
$$

The solid lines in Fig. 2 denote the results of the curve fitting. In the higher $q$-region $\left(q>0.1 \AA^{-1}\right)$, a discrepancy between the observed SAXS intensities and the fitted curves is apparent, which is likely due to scattering from heterogeneity in the core portion of the micelles. Hence, we used the SAXS data sets in the lower $q$-range of 0.018 $0.1 \AA^{-1}$ for the model fitting analysis.

The fitted parameters are listed in Table 1. For $C_{\mathrm{PGPR}}=$ $18.35 \mathrm{wt} \%\left(\phi_{\mathrm{PGPR}}=0.185\right)$, the fitted values were $\langle R>=$ $29.7 \AA, \sigma=0.4<R>$, and $\phi=0.185$. Figure 4 shows the structure factor, $S_{\mathrm{PY}}(q)$, which indicates that inter-particle interference increases significantly with increasing $C_{\mathrm{PGPR}}$. The PGPRs had various molecular weights, from 727 to 1394, causing the microemulsions formed by the PGPR to show a wide distribution in their radius size. Figure 5 shows the $C_{\mathrm{PGPR}}$ dependence of the mean radius of the PGPR micelles, $<R_{\mathrm{M}}>$. The $<R_{\mathrm{M}}>$ value is rather constant irrespective of $C_{\mathrm{PGPR}}$. This is reasonable given that these samples were simply prepared by dilution and the micelle size is not sensitive to the concentration but is determined by the chemical structure of PGPR.

\subsection{Vitamin E encapsulating PGPR emulsions}

The SAXS measurements were also conducted for mixtures of PGPR and vitamin E in glycerol. The PGPR concentration was fixed at $17.85 \mathrm{wt} \%$ and vitamin $\mathrm{E}$ was systematically added to the solution. Figure 6 shows the SAXS curves for the mixtures, where vitamin $\mathrm{E}$ concentrations, $C_{\mathrm{VE}}$, were $0,0.27,0.675,2.7$, and $3.38 \mathrm{wt} \%$. As shown in Fig. 6, the scattering intensity increases and the scattering maximum shifts towards a lower $q$ value with increasing $C_{\mathrm{VE}}$.

Figure 7 shows the SLD variations of the vitamin E mi-

Table 1 The values of fitting parameter obtained from SAXS measurements for PGPR solutions.

\begin{tabular}{lcccccc}
\hline \multicolumn{1}{c}{$C_{\mathrm{PGPR}} / \mathrm{wt} \%$} & 1.00 & 2.50 & 5.00 & 10.00 & 15.00 & 18.35 \\
\hline$R_{\mathrm{M}} / \AA$ & 22.57 & 26.64 & 26.17 & 27.48 & 30.35 & 29.68 \\
$\phi$ & 0.0101 & 0.0252 & 0.0503 & 0.1006 & 0.1509 & 0.1846 \\
$\sigma / \mathrm{R}$ & 0.4 & 0.4 & 0.4 & 0.4 & 0.4 & 0.4 \\
\hline
\end{tabular}

Uncertainties of all fitting parameters were less than $1 \%$. 


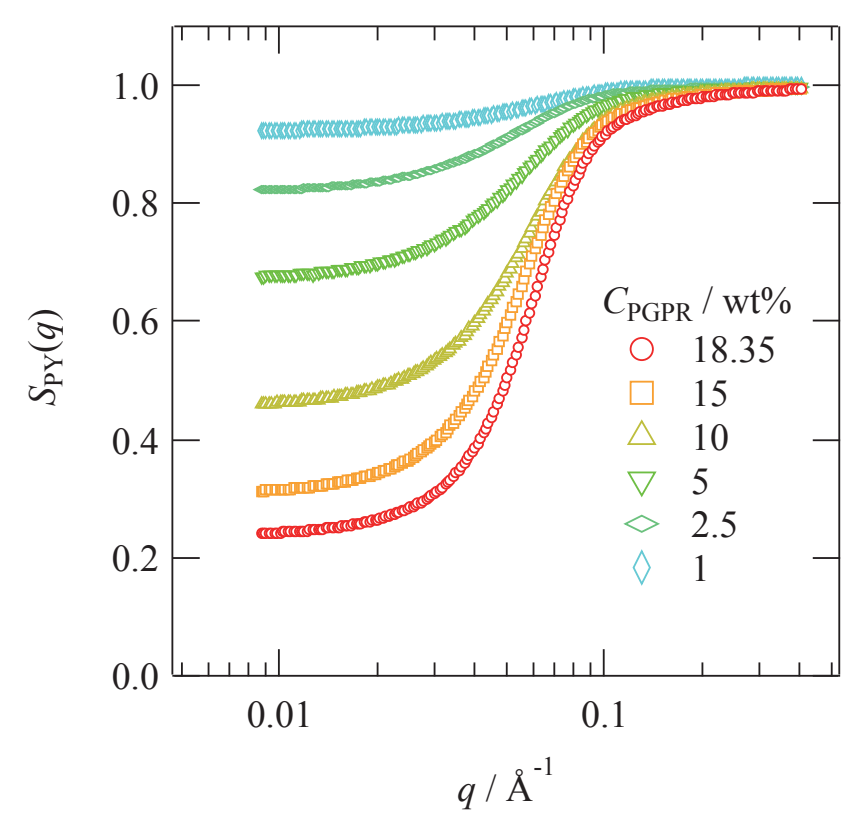

Fig. 4 Inter-particle interference function, $S_{\mathrm{PY}}(q)$, used for the curve fitting of the SAXS functions of PGPR micelles.

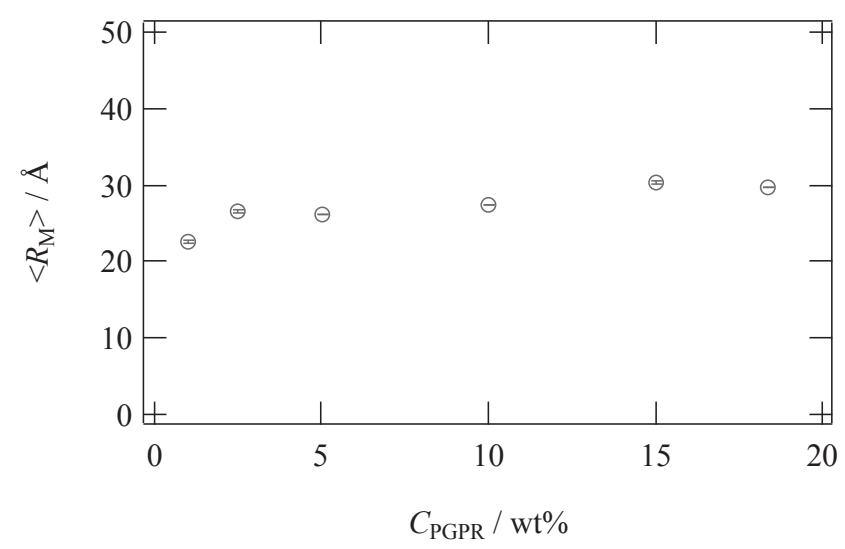

Fig. $5 C_{\mathrm{PGPR}}$ dependence of the mean radius of PGPR micelles, $<R_{\mathrm{M}}>$.

croemulsion $\left(d_{\mathrm{VE}}=0.950 \mathrm{~g} / \mathrm{cm}^{3}\right)$. The SLD of the head group of the PGPR molecule is very close to that of glycerol and the SLD of the hydrophobic group of the PGPR molecule is very close to that of vitamin $\mathrm{E}$. We performed curve fitting of the observed SAXS intensity functions by using a spherical model with box-type SLD variation. The upticks in the observed SAXS intensity for $C_{\mathrm{VE}}=0.27 \mathrm{wt} \%$ in the lower $q$ region can be attributed to scattering from a partial aggregate of the vitamin E microemulsions. Hence, we ignored this region in the fitting. The SAXS data sets in the $q$-range of $0.018-0.11 \AA^{-1}$ were used for the model fitting analysis of the vitamin $\mathrm{E}$ microemulsion.

The fitted results for $C_{\mathrm{VE}}=2.7 \mathrm{wt} \%$ are $\langle R>=74.0 \AA$, $\sigma$ $=0.4<R>$, and $\phi=0.214$. The solid lines in Fig. 6 are the fit determined using the same equations shown above, and

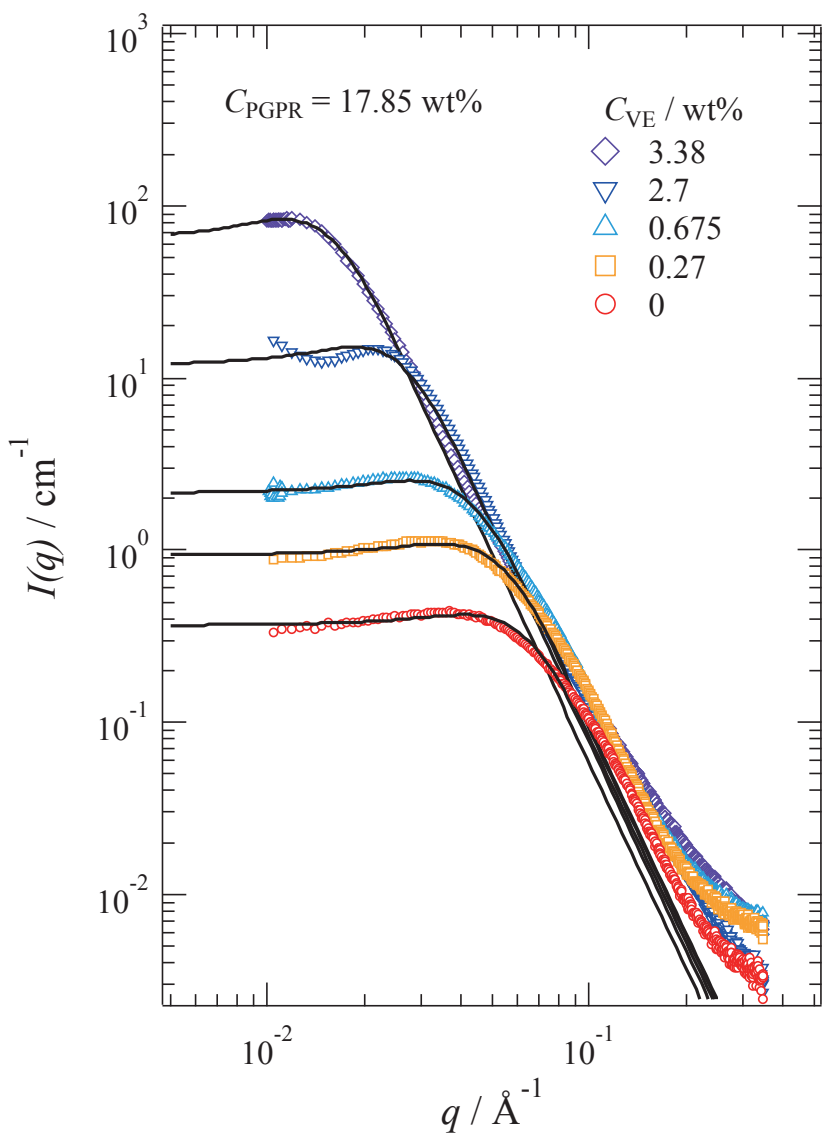

Fig. 6 SAXS curves of vitamin $\mathrm{E}$ microemulsions in glycerol at various Vitamin E concentrations, $C_{\mathrm{VE}}$. The solid lines are fitting results with Eq. (2)-(6).

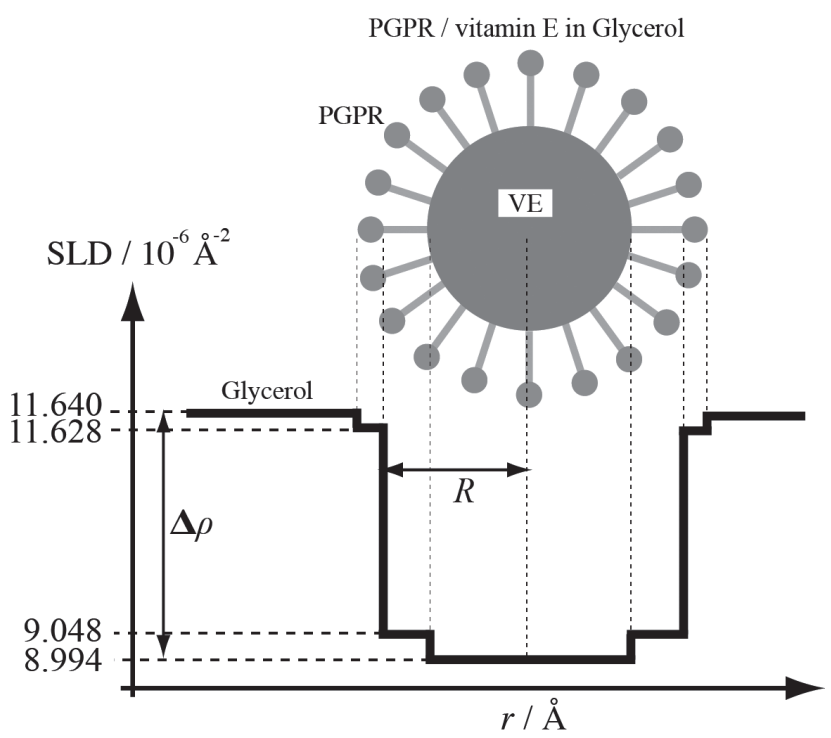

Fig. 7 The calculated scattering length density profile of vitamin $\mathrm{E}$ microemulsion in glycerol. 


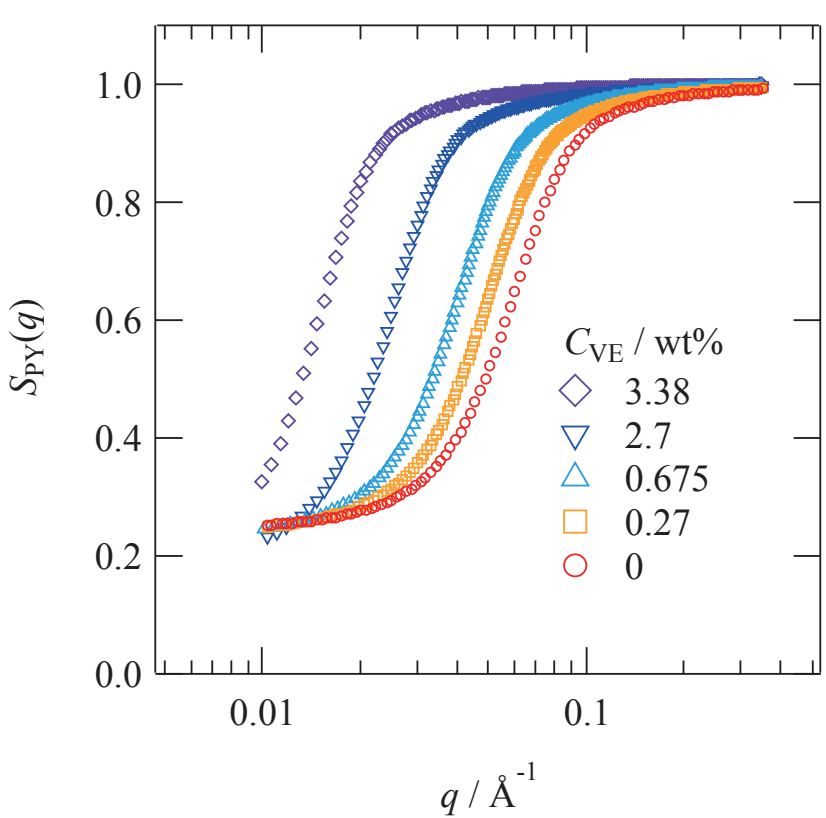

Fig. 8 Inter-particle interference function, $S_{\mathrm{PY}}(q)$, used for the curve fitting of the SAXS functions of PGPR micelles.

the fitting seems to be satisfactory. The upticks in the observed SAXS intensity functions at high $q$ values can be attributed to scattering from local structures of the PGPR. Hence, we ignored this region in the fitting. Figure 8 shows the structure factor, $S_{\mathrm{PY}}(q)$. The inter-particle interaction further increases with the incorporation of increasing amounts of vitamin $\mathrm{E}$.

Figure 9 shows the $C_{\mathrm{VE}}$ dependence of the mean radius of the VE microemulsion, $\left\langle R_{\mathrm{ME}}>\right.$. The core radius of microemulsions containing vitamin $\mathrm{E}$ core, $R_{\text {core }}$, is defined by

$$
R_{\text {core }}=R_{\mathrm{ME}}-R_{\mathrm{M}}
$$

where $R_{\mathrm{ME}}$ is the radius of the microemulsion, and $R_{\mathrm{M}}$ is the radius of the PGPR micelle $\left(C_{\mathrm{VE}}=0 \mathrm{wt} \%\right) . R_{\text {core }}$ is related to the number of vitamin $\mathrm{E}$ molecules in a microemulsion, $n_{\mathrm{VE}}$, by

$$
\begin{aligned}
n_{\mathrm{VE}}= & \frac{4 \pi R_{\text {core }}^{3}}{3 v_{\mathrm{VE}}}, \quad n_{\mathrm{PGPR}}=\frac{4 \pi R_{\text {core }}^{2}}{s_{\mathrm{PGPR}}}, \\
& \frac{n_{\mathrm{VE}}}{n_{\mathrm{PGPR}}}=\frac{\left(4 \pi R_{\text {core }}^{3} / 3 v_{\mathrm{VE}}\right)}{\left(4 \pi R_{\text {core }}^{2} / s_{\mathrm{PGPR}}\right)}=\frac{s_{\mathrm{PGPR}}}{3 v_{\mathrm{VE}}} R_{\text {core }}
\end{aligned}
$$

Here, $v_{\mathrm{VE}}$ is the volume of the vitamin E molecule, and $n_{\mathrm{PGPR}}$ is the number of PGPR molecules in a single emulsion. Hence,

$$
<R_{\mathrm{ME}}>\sim R_{\text {core }}=\frac{3 v_{\mathrm{VE}} n_{\mathrm{VE}}}{s_{\mathrm{PGPR}} n_{\mathrm{PGPR}}} \sim \frac{C_{\mathrm{VE}}}{C_{\mathrm{PGPR}}} C_{\mathrm{VE}}
$$

As observed in Fig. 9, $<R_{\mathrm{ME}}>$ linearly increases with increasing $C_{\mathrm{VE}}$, which indicated the formation of a microemulsion containing vitamin $\mathrm{E}$.

The zero-angle scattering intensity $I(0)$ is proportional to the number of scattering objects, described by the follow-

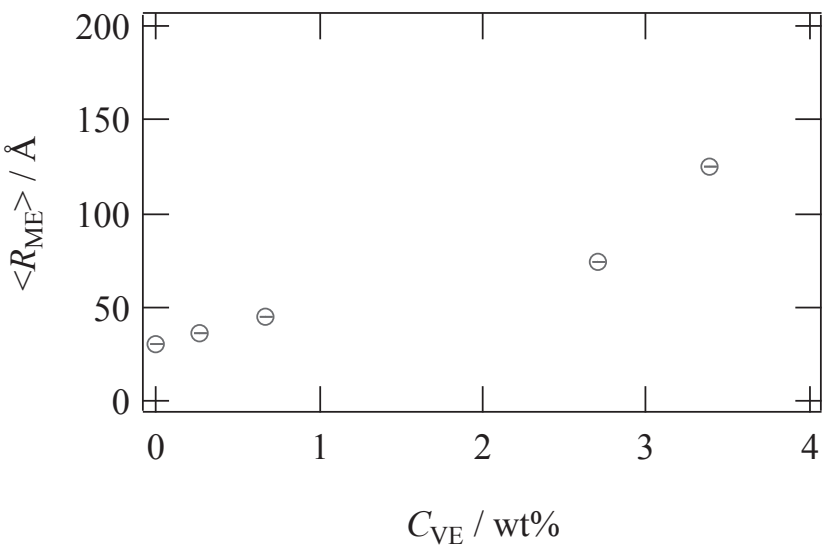

Fig. $9 C_{\mathrm{VE}}$ dependence of the mean radius of microemulsion, $<R_{\mathrm{ME}}>$.

ing relation,

$$
I(0)=N V^{2}<\Delta \rho>^{2}
$$

where $N$ is the number density of the scattering objects. In general, surfactants possess a critical micelle concentration (cmc) where they start to form micelles. To estimate the number of PGPR molecules in a micelle, we assume here that the same number of PGPR molecules, $N_{\text {PGPR }}$, participates in the formation of micelles (M; PGPR only) and of microemulsions (ME; PGPR + vitamin E). Since the PGPR concentration is constant, the following equation can be used,

$$
N_{\mathrm{PGPR}}=N_{M} \frac{S_{M}}{S_{\mathrm{PGPR}}}=N_{M E} \frac{S_{M E}}{S_{\mathrm{PGPR}}}
$$

where $N_{\mathrm{M}}$ and $N_{\mathrm{ME}}$ are the number of micelles and microemulsions in the system, respectively, $S_{\mathrm{M}}$ and $S_{\mathrm{ME}}$ are the surface area of a single micelle or microemulsion, respectively, and $s_{\mathrm{PGPR}}$ denotes the surface coverage area of the PGPR molecule (the surface area occupied by single PGPR molecule) on a micelle or a microemulsion. Hence, $N_{\mathrm{i}}$ and $S_{\mathrm{i}}$ ( $i=\mathrm{M}$ or $\mathrm{ME}$ ) are inversely proportional to each other, i.e.,

$$
\frac{N_{M E}}{N_{M}}=\frac{S_{M}}{S_{M E}}
$$

We estimated the ratio of $N_{\mathrm{ME}} / N_{\mathrm{M}}$ from $I(0)$ values and the ratio of $S_{\mathrm{M}} / S_{\mathrm{ME}}$ from the radius data, i.e., $\left\langle R_{\mathrm{M}}>\right.$ and $<R_{\mathrm{ME}}>$. The ratios are 0.18 and 0.16 , respectively, and are in good agreement to Eq. (12). This supports the hypothesis that the same number of PGPR molecules participate in formation of micelles and microemulsions. We also estimated the number of vitamin E molecules, $n_{\mathrm{VE}}$, encapsulating PGPR emulsions from SAXS results by using Eq. (8). Figure 10 shows the $C_{\mathrm{VE}}$ dependence of $n_{\mathrm{VE}}$ showing that the value of $n_{\mathrm{VE}}$ increases with $C_{\mathrm{VE}}$. From these results, it can be concluded that a large number of vitamin $\mathrm{E}$ molecules can be stably encapsulated by a PGPR microemulsion. 


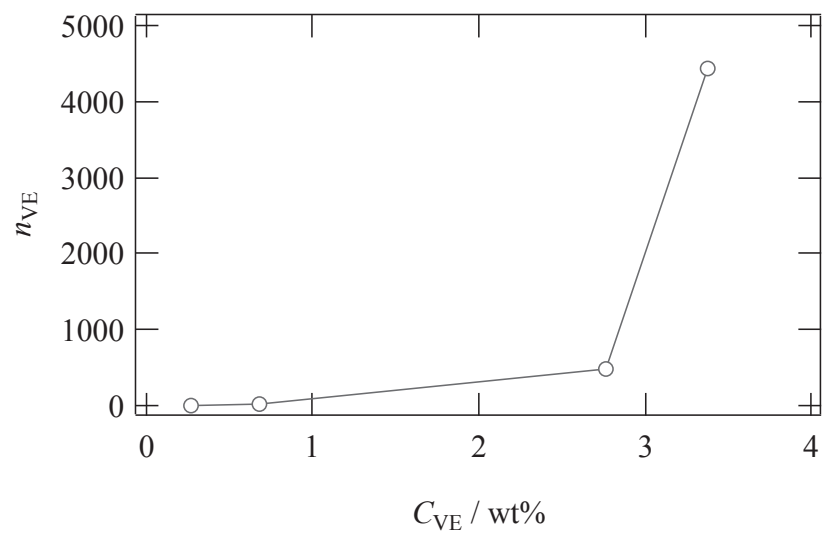

Fig. $10 C_{\mathrm{VE}}$ dependence of the number of vitamin $\mathrm{E}$ in a microemulsion, $n_{\mathrm{VE}}$.

\subsection{Rheological behavior of Vitamin E encapsulating PGPR emulsions}

Figure 11 shows the viscosity as a function of shear rate for glycerol, PGPR micelles, and vitamin E microemulsions. Note that the viscosity measurements were conducted during both the increasing and decreasing processes of the shear rate. The viscosity of the PGPR solutions were much higher than that of pure glycerol (solvent). This is due to the formation of PGPR micelles, as observed by SAXS. The high viscosity behavior of PGPR solutions is due to high PGPR concentration ( $\phi=0.185$ ), enough to generate significant inter-particle interaction. In fact, SAXS profiles for the PGPR solution $\left(C_{\mathrm{VE}}=0 \mathrm{wt} \%\right)$ exhibited a peak originating from inter-particle interaction. The viscosity of the mixtures of PGPR and vitamin $\mathrm{E}\left(C_{\mathrm{VE}}=2.7 \mathrm{wt} \%\right)$ was higher than that of the PGPR solution. This result indicates the formation of vitamin $\mathrm{E}$ microemulsions stabilized by PGPR, which is consistent with the SAXS results. Furthermore, the mixtures of PGPR and vitamin E show shear thinning behavior, which is likely due to the destruction of the microemulsions. The viscosity data shows little hysteresis in the process of decreasing shear rate, indicating the stable formation of microemulsions and that the microemulsions are able to recover to a stable steady state after shearing.

\section{Conclusion}

There are a limited number of surfactants available for foods and food ingredients, and PGPR is known to be one of the best for emulsifying food additives. Here, we investigated the structures of PGPR micelles and their derivative microemulsions. Figure 12 schematically illustrates PGPR micelle and vitamin E microemulsion, respectively. From the SAXS results, the size of PGPR micelles in glycerol was determined to be ca. $30 \AA$. When, vitamin $\mathrm{E}$ was added to the micelles, a spontaneous increase in size was observed to form microemulsions The mean radius of the microemul-

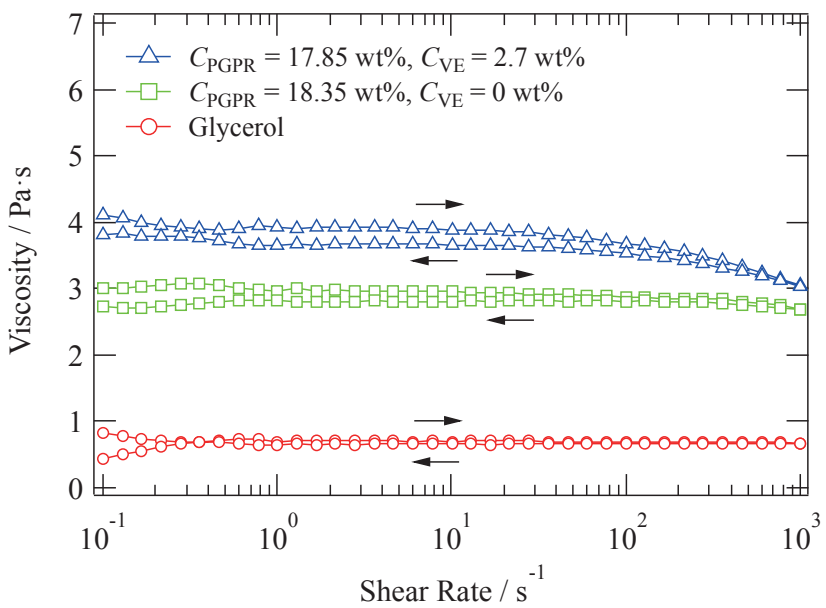

Fig. 11 Shear rate dependence of the viscosities for glycerol, PGPR micelles, and vitamin E microemulsions.

PGPR in Glycerol

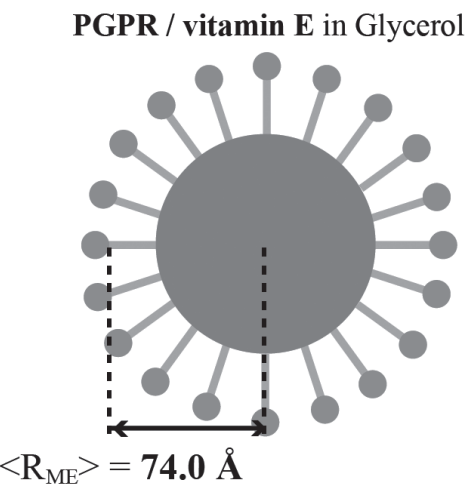

Fig. 12 Schematic illustration of PGPR micelle and vitamin $\mathrm{E}$ microemulsion.

sion increased linearly with vitamin $\mathrm{E}$ concentration, indicating the stable encapsulation of vitamin E. Furthermore, the ratio of scattering intensity between PGPR micelles and vitamin $\mathrm{E}$ microemulsions was equivalent to the value calculated by the assumption that there are vitamin $\mathrm{E}$ emulsions stabilized by PGPR. These results are also supported by the rheological measurements. The micelles were stable against shearing up to the shear rate of $1000 \mathrm{~s}^{-1}$. In addition, shear thinning occurred in the emulsion above $100 \mathrm{~s}^{-1}$, indicating that the emulsion may undergo a break up by shearing. However, once the shear was reduced, the microemulsions recovered to a stable steady state, which indicates high stability and resilience of the microemulsions.

\section{Acknowledgment}

The SAXS experiment was conducted at the second hutch of the Frontier Soft Matter Beamline(FSBL; 
BL03XU), SPring-8, Hyogo, Japan, with the assistance of Atsushi Izumi, Sumitomo Bakelite, Co., Ltd. with proposal no. 2014B7260. The SAXS experiment was also performed with the approval of the Japan Synchrotron Radiation Research Institute (JASRI) (Proposal No. 2014A1815).

\section{References}

1) Kim, C. J.; Kurauchi, S.; Uebayashi, T.; Fujisaki, A.; Kimura, S. Morphology change from nanotube to vesicle and monolayer/bilayer alteration by amphiphilic block polypeptides having aromatic groups at C terminal. Bull. Chem. Soc. Jpn. 90, 568-573(2017).

2) Yu, C.; Zhou, W.; Liu, H.; Liu, Y.; Dionysiou, D.D. Design and fabrication of microsphere photocatalysts for environmental purification and energy conversion. Chem. Eng. J. 287, 117-129(2016).

3) Nawa, E.; Yamamoto, D.; Shioi, A. Chemotactic amoeboid-like shape change of a vesicle under a $\mathrm{pH}$ gradient. Bull. Chem. Soc. Jpn. 88, 1536-1544(2015).

4) Ramanathan, M.; Shrestha, L.K.; Mori, T.; Ji, Q.; Hill, J.P.; Ariga, K. Amphiphile nanoarchitectonics: from basic physical chemistry to advanced applications. Phys. Chem. Chem. Phys. 15, 10580-10611 (2013).

5) Flanagan, J.; Harjinder, S. Microemulsions: a potential delivery system for bioactives in food. Crit. Rev. Food Sci. Nutr. 46, 221-237(2006).

6) McClements, D.J. Food Emulsions, Principles, Practice, and Techniques. 2nd ed. CRC Press, Boca Raton, pp. 137-144(2004).

7) Katsuragi, T.; Ishitobi, M. Polyglycerol fatty acid esters: Effects of structure and composition on functions. Foods Food Ingred. J. Jpn. 180, 35-44 (1999).

8) Wakisaka, S.; Nakanishi, M.; Gohtani, S. Phase behavior and formation of $\mathrm{O} / \mathrm{W}$ nano-emulsion in vegetable oil/mixture of polyglycerol polyricinoleate and polyglycerin fatty acid ester/water systems. J. Oleo Sci. 63, 229-237 (2014).

9) Julianto, T.; Yuen, K.; Noor, A. Improved bioavailability of vitamin $\mathrm{E}$ with a self emulsifying formulation. Int. J. Pharm. 200, 53-57 (2000).

10) Huang, S.-W.; Frankel, E.N.; German, J.B. Antioxidant activity of alpha- and gamma-tocopherols in bulk oils and in oil-in-water emulsions. J. Agric. Food Chem. 42, 2108-2114 (1994).

11) Paul, B.K.; Moulik, S.P. Microemulsions: an overview. J. Disp. Sci. Technol. 18, 301-367(1997).

12) Regev, O.; Ezrahi, S.; Aserin, A.; Garti, N.; Wachtel, E.; Kaler, E. W.; Khan, A.; Talmon, Y. A study of the microstructure of a four-component nonionic microemulsion by Cryo-TEM, NMR, SAXS, and SANS. Langmuir 12, 668-674 (1996).

13) Yaghmur, A.; de Campo, L.; Aserin, A.; Garti, N.; Glatter, O. Structural characterization of five-component food grade oil-in-water nonionic microemulsions. Phys. Chem. Chem. Phys. 6, 1524-1533(2004).

14) Jestin, J.; Simon, S.; Zupancic, L.; Barre, L. A small angle neutron scattering study of the adsorbed asphaltene layer in water-in-hydrocarbon emulsions: Structural description related to stability. Langmuir 23, 10471-10478(2007).

15) Nakamura, T.; Aoi, N. Jpn. Pat. Kokai 1995-100355 (1995.4.18).

16) Zhang, F.; Ilavsky, J.; Long, G.G.; Quintana, J.P.G.; Allen, A.J.; Jemian, P.R. Glassy carbon as an absolute intensity calibration standard for small-angle scattering. Metall. Mater. Trans. A 41A, 1151-1158(2010).

17) Percus, J.K.; Yevick, G.J. Analysis of classical statistical mechanics by means of collective coordinates. Phys. Rev. 110, 1-12(1958). 